\title{
Risk of Clostridium difficile diarrhea among hospital inpatients prescribed proton pump inhibitors: cohort and case-control studies
}

\author{
Sandra Dial, Khalid Alrasadi, Chantal Manoukian, Allen Huang, Dick Menzies \\ Fast-tracked article. Published at www.cmaj.ca on June 4, 2004.
}

ß See related articles pages 19, 27, 45, 47 and 51

\section{Abstract}

Background: Antibiotic disruption of the normal intestinal flora is a well-known risk factor for Clostridium difficile-associated diarrhea. Reduced gastric acidity has been suggested as a risk factor, and we hypothesized that proton pump inhibitors, because of their potency, may be an independent risk factor for this problem.

Methods: For the cohort study we identified from a pharmacy database 1187 inpatients at a Montreal teaching hospital who received antibiotics over a 9-month period beginning in August 2002. We compared patients in this group who had also received a proton pump inhibitor or an $\mathrm{H}_{2}$ blocker with patients who had not received acid suppressive therapy. Hospital laboratory reports of positive assay results for $C$. difficile toxin were used to ascertain cases in the cohort.

To assess the possibility that proton pump inhibitors were prescribed to patients who were sicker and had other risk factors for $C$. difficile infection, we did a case-control study at a second Montreal teaching hospital. Cases were defined as patients who were positive for $C$. difficile toxin and who had a history of diarrhea $(n=94)$. Control subjects were selected from among patients who had received an antibiotic and were matched to cases by ward, age within 5 years and class of antibiotics $(n=94)$.

Results: In the cohort study, $C$. difficile diarrhea developed in 81 $(6.8 \%)$ of the 1187 patients who received antibiotics while in hospital. In a multivariate analysis, $C$. difficile diarrhea was significantly associated with use of proton pump inhibitors (adjusted odds ratio [OR] 2.1, 95\% confidence interval [CI] 1.2-3.5), receipt of 3 or more antibiotics (OR 2.1, 95\% Cl 1.33.4) and admission to a medical ward (OR 4.1, 95\% Cl 2.37.3). In the case-control study $C$. difficile diarrhea was associated with female sex (adjusted OR 2.1, 95\% Cl 1.1-4.0), prior renal failure (adjusted OR 4.3, 95\% Cl 1.5-11.9), hospital admission in the 3 months before the index admission (adjusted OR 2.6, 95\% Cl 1.4-5.2) and use of proton pump inhibitors (adjusted OR 2.7, 95\% Cl 1.4-5.2).

Interpretation: Patients in hospital who received proton pump inhibitors were at increased risk of $C$. difficile diarrhea.

CMAI 2004;171(1):33-8

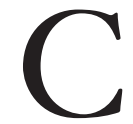
lostridium difficile is the most common cause of nosocomial infectious diarrhea in industrialized countries ${ }^{1}$ and has been reported to be increasing in frequency and severity, 2,3 with estimated health care costs of over $\$ 1.1$ billion in the United States each year. ${ }^{4}$ The main cause of this problem is believed to be antibiotic disruption of the normal intestinal flora, resulting in overgrowth of $C$. difficile. Use of proton pump inhibitors has been associated with colonization of the normally sterile upper gastrointestinal tract ${ }^{6}$ and can therefore alter gastrointestinal flora. Decreased gastric acidity is a known risk factor for other infectious diarrheal illnesses such as travellers' diarrhea, salmonellosis and cholera, ${ }^{7}$ and because the survival of $C$. difficile and its toxin are facilitated by higher gastric $\mathrm{pH}$ levels, ${ }^{8}$ it may also be a risk factor for $C$. difficile diarrhea. This idea is supported by reports of a possible association between $C$. difficile diarrhea and acid suppressive therapy, ${ }^{3,4,-11}$ and case reports of $C$. difficile diarrhea in patients with pernicious anemia ${ }^{8}$ and in patients receiving Helicobacter pylori treatment, which combines gastric acid suppressive therapy with antibiotics. ${ }^{12,13}$

Proton pump inhibitors are potent inhibitors of gastric acid production. ${ }^{14}$ These drugs have been proven effective in many indications, and as a class these agents had the largest increase in unit sales in North America in 2002. We have observed an increase in the incidence of $C$. difficile diarrhea, coincident with increased use of proton pump inhibitors. To address this possible association, we examined the risk of $C$. difficile-associated diarrhea in hospital patients who were receiving proton pump inhibitors.

\section{Methods}

\section{Cohort study}

We defined the cohort as all patients identified from a pharmacy database who had received antibiotics between Aug. 1, 2002, and Apr. 30, 2003, on the 2 general medical wards and the cardiothoracic surgical ward at the Royal Victoria Hospital, a Montréal teaching hospital. These 3 wards were chosen because they had all experienced significant increases in the incidence of $C$. difficile disease compared with the previous year. Because the patients were 
identified from a pharmacy database, the only information available for analysis was on medications, the ward, the total number and type of antibiotics, and the type of acid suppressive therapy (e.g., proton pump inhibitor or $\mathrm{H}_{2}$ blocker). Cohort patients with $C$. difficile infection were identified by verifying if their names appeared in a registry of patients with a positive toxin assay result, maintained by the hospital's infection control service. Because hospital policy requires the clinical laboratory to report all positive toxin assay results to this registry, we assumed that cohort patients whose names were not in the registry had not had $C$. difficile infection.

\section{Case-control study}

Because the data available from the cohort study was limited and because we wanted to address the possibility that proton pump inhibitors were prescribed to patients who were sicker and had other risk factors for $C$. difficile colitis, we performed a case-control study at a second Montréal teaching hospital (the Sir Mortimer B. Davis Jewish General Hospital) during the same study period. Cases were defined as all consecutive patients on all wards in the hospital who had a history of diarrhea (defined as 2 or more loose bowel movements per day) and a positive $C$. difficile toxin assay result from a stool sample. Because our objective was to study new hospital-acquired cases, we included only patients who had never been diagnosed with $C$. difficile diarrhea previously and whose first positive toxin assay result was reported during or within 1 month after their index hospital admission.

Control subjects were selected from a list obtained from the hospital pharmacy of patients who had been prescribed any antibiotics while in hospital during the study period. To control for other risk factors previously associated with an increased risk of $C$. difficile diarrhea, control subjects were frequency matched to the cases by inpatient ward, ${ }^{15}$ age within 5 years, class of antibiotics (in particular quinolones, cephalosporins [first-generation, or second- and third-generation], penicillins, carbapenems and macrolides) and, if possible, number of antibiotics. ${ }^{16,17}$ To ensure adequate time of exposure, and equal opportunity for ascertainment, we considered control subjects eligible if they had been in hospital for at least 5 days and had survived at least 30 days from the time of hospital admission.

\section{Proton pump inhibitor exposure}

To be considered exposed, patients had to have received these drugs for at least 3 days before diarrhea developed. For patients who did not have diarrhea, this therapy had to have been prescribed for at least 3 days in hospital. Long-term use was defined as use of these drugs for more than 6 months before the development of $C$. difficile diarrhea.

\section{Data collection}

A standardized form was used to abstract data from the medical records of the cases and control subjects. The following information was collected: age, sex, any institutionalization (defined as long-term residence in a chronic care setting or hospital admission in an acute care setting for more than 2 months), prior hospital admission or antibiotic use in the 3 months before the index hospital admission, diagnosis on admission, and comorbid illnesses, particularly diabetes mellitus, renal failure (defined as a creatinine level greater than $250 \mathrm{mmol} / \mathrm{L}$ ), peptic ulcer disease, gastroesophageal reflux disease, cancer and pernicious anemia. Charlson Comorbidity Index scores ${ }^{18}$ were calculated for the case and control subjects. Information was collected on all medications taken in the 30 days before the diagnosis of $C$. difficile diarrhea (cases) or during the hospital stay (controls). The indication for acid suppressive therapy was also recorded. Outcomes ascertained included surgical colectomy, relapses, admission to the intensive care unit (ICU), acute renal failure requiring dialysis and death within 30 days after the development of C. difficile diarrhea. A relapse was defined as a recurrent episode of diarrhea with a positive C. difficile toxin assay result after completion of treatment for C. difficile and resolution of diarrhea.

At both hospitals tissue culture cytotoxic assays were performed with diarrheal stool samples using the C. difficile Toxin/ Antitoxin Kit (TechLab Inc., Blacksburg, Va.). ${ }^{19}$

The studies were approved by the Ethics Review Board of the McGill University Health Centre for the cohort study and by the Jewish General Hospital Ethics Board for the case-control study.

\section{Data analysis}

In the cohort study, the relative risks for the development of C. difficile diarrhea in association with the use of proton pump inhibitors, $\mathrm{H}_{2}$ blockers and antibiotics, taken separately and in various combinations, were estimated along with the corresponding $95 \%$ confidence intervals (CIs). ${ }^{20}$ Multivariate logistic regression was used to obtain the adjusted odds ratio (OR) of the effect of exposure to proton pump inhibitors after adjustment for the confounders of exposure to 3 or more antibiotics and type of ward.

In the case-control study, characteristics of the case and control subjects were compared and tested for significant differences using a Student $t$ test for linear variables and a $\chi^{2}$ test for categorical variables. For the primary analysis of risk factors associated with $C$. difficile diarrhea, ORs and $95 \%$ CIs of possible risk factors were estimated, and a multivariate logistic regression was performed to adjust for potentially confounding factors. Variables were included in the multivariate model if the univariate analysis showed that they were significantly associated with $C$. diarrbea or showed evidence of a substantial effect; also included were clinical factors associated with $C$. difficile diarrhea in previous studies. We evaluated 2 separate regression models; methicillin-resistant Staphylococcus aureus (MRSA) colonization was excluded from the first model because of a concern of detection bias, but this variable was included in the second model.

\section{Results}

\section{Cohort study}

Between Aug. 1, 2002, and Apr. 30, 2003, 1187 patients were prescribed antibiotics while in hospital on 1 of the 3 wards studied. In total, $C$. difficile diarrhea developed in 81 (6.8\%) of the patients: 55 (9.3\%) of the 591 patients who also received proton pump inhibitors and 26 (4.4\%) of the $596 \mathrm{pa}-$ tients who did not receive these drugs. Table 1 shows the incidence of $C$. difficile diarrhea among patients with different ward and antibiotic exposures, including those receiving only 1 antibiotic, and the relative risk associated with proton pump 
inhibitor use. For many of the risk factors examined, the relative risk of $C$. difficile diarrhea was higher among the patients who were prescribed proton pump inhibitors than among those who were not prescribed these drugs.

C. difficile diarrhea developed in $10.9 \%$ of the cohort patients on the medical wards, as compared with $2.9 \%$ of those on the surgical ward $(p<0.001)$, and in $12.1 \%$ of the patients who received 3 or more antibiotics, as compared with $5.1 \%$ of those who received only 1 or 2 different antibiotics $(p<0.001)$. Among the 354 patients exposed to high-risk antibiotics ${ }^{21}$ (clindamycin, second- or thirdgeneration cephalosporins, or ampicillin or its analogues), C. difficile diarrhea developed in 30 (8.5\%), as compared with $51(6.1 \%)$ of the 833 patients given other antibiotics. Patients on the surgical ward were less likely than those on the medical wards to be exposed to 3 or more antibiotics or to high-risk antibiotics (data not shown).

After adjustment in the multivariate analysis for the number of antibiotics received and the ward, C. difficile diarrhea remained significantly associated with the use of proton pump inhibitors (OR 2.1, 95\% CI 1.2-3.5) but not with the use of $\mathrm{H}_{2}$ blockers (Table 2).

\section{Case-control study}

Ninety-four patients met the case definition for $C$. difficile diarrhea. As seen in Table 3, the case and control subjects were similar in age, number and type of antibiotics, and Charlson Comorbidity Index scores. However, the cases were more likely than the control subjects to be female, to have had renal failure, to have MRSA colonization and to have been admitted to hospital in the 3 months before the index admission. The cases had significantly increased morbidity and mortality: 18 required admission to the ICU with fulminant $C$. difficile as the admitting diagnosis, 12 experienced acute renal failure requiring dialysis, 8 had total colectomies, and 21 died within 30 days after the diagnosis of $C$. difficile diarrhea. Of the cases, 60 (64\%) were receiving proton pump inhibitors, as compared with $34(36 \%)$ of the control subjects (unadjusted OR 3.1, 95\% CI 1.7-5.6). Interestingly, C. difficile diarrhea developed in a patient receiving a proton pump inhibitor who was not taking an antibiotic. The cases were also more likely than the control subjects to have had prolonged exposure ( $>6$ months) to a proton pump inhibitor.

After adjusting for all other significant factors, we found that use of proton pump inhibitors was significantly associated with $C$. difficile diarrhea (Table 4). The association of

Table 2: Factors associated with $C$. difficile diarrhea in cohort of 1187 patients given antibiotics while in hospital*

\begin{tabular}{lcc}
\hline Factor & $\begin{array}{c}\text { Unadjusted OR } \\
(95 \% \mathrm{Cl})\end{array}$ & $\begin{array}{c}\text { Adjusted OR } \\
(95 \% \mathrm{Cl})\end{array}$ \\
\hline $\begin{array}{l}\text { PPI use (v. no acid suppressive } \\
\text { therapy) }\end{array}$ & $2.1(1.4-3.4)$ & $2.1(1.2-3.5)$ \\
$\begin{array}{l}\mathrm{H}_{2} \text { blocker (v. no acid suppressive } \\
\text { therapy) }\end{array}$ & $0.4(0.1-1.2)$ & $1.1(0.4-3.4)$ \\
$\geq 3$ antibiotics $(\mathrm{v} .<3)$ & $2.4(1.6-3.6)$ & $2.1(1.3-3.4)$ \\
Medical ward (v. surgical ward) & $4.5(2.6-8.3)$ & $4.1(2.3-7.3)$ \\
\hline
\end{tabular}

Note: $\mathrm{OR}=$ odds ratio

*The variable "high-risk antibiotic" (defined in Table 1) was not included in the final model because it was significantly confounded by type of ward.

\section{Table 1: Relative risk of Clostridium difficile diarrhea in relation to use of proton pump} inhibitors (PPIs) in cohort of 1187 patients who received antibiotics while in hospital

\begin{tabular}{|c|c|c|c|}
\hline \multirow[b]{2}{*}{ Variable } & \multicolumn{2}{|c|}{$\begin{array}{l}\text { No. of cases of diarrhea/ } \\
\text { total no. with risk factor }(\%)\end{array}$} & \multirow[b]{2}{*}{$\mathrm{RR}(95 \% \mathrm{Cl})$} \\
\hline & Patients taking PPIs & Patients not taking PPIs & \\
\hline $\begin{array}{l}\text { Total no. of cases of } \\
\text { C. difficile diarrhea }\end{array}$ & $55 / 591 \quad(9.3)$ & $26 / 596 \quad(4.4)$ & $2.1(1.4-3.4)$ \\
\hline \multicolumn{4}{|l|}{ Ward } \\
\hline Surgical & $13 / 287 \quad(4.5)$ & $4 / 301 \quad(1.3)$ & $3.4(1.1-10.3)$ \\
\hline Medical & $42 / 294(14.3)$ & $22 / 295 \quad(7.5)$ & $1.9(1.2-3.1)$ \\
\hline \multicolumn{4}{|l|}{ Antibiotic exposure } \\
\hline 1 antibiotic & $24 / 261 \quad(9.2)$ & $10 / 333 \quad(3.0)$ & $3.1(1.5-6.3)$ \\
\hline 2 antibiotics & $8 / 146 \quad(5.5)$ & $3 / 150 \quad(2.0)$ & $2.8(0.8-10.3)$ \\
\hline$\geq 3$ antibiotics & 23/184 (12.5) & $13 / 113(11.5)$ & $1.1(0.6-2.1)$ \\
\hline High-risk antibiotic* & 16/181 (8.8) & $14 / 173 \quad(8.1)$ & $1.1(0.6-2.2)$ \\
\hline \multicolumn{4}{|l|}{ Single-use antibiotic } \\
\hline Cefazolin & $6 / 54 \quad(11.1)$ & $(1.2)$ & $9.2(1.1-74.1)$ \\
\hline Any quinolone† & $(19.1)$ & $3 / 44$ & $2.8(0.8-9.7)$ \\
\hline Vancomycin & $(3.3)$ & 2/103 (1.9) & $1.7(0.3-9.9)$ \\
\hline $\begin{array}{l}\text { Any second- or third- } \\
\text { generation cephalosporin }\end{array}$ & $(8.3)$ & $(7.7)$ & $1.1(0.1-15.5)$ \\
\hline
\end{tabular}


MRSA colonization with $C$. difficile, seen in Table 3, may have resulted from detection bias, because all patients with positive $C$. difficile toxin assay results were also routinely screened for MRSA. Therefore, we initially excluded MRSA status from the multivariate model. However, even when we included it, we found that it did not significantly alter the association between proton pump inhibitor use and $C$. difficile diarrhea (Table 4).
Of the 21 patients who had one or more relapses, 19 (90\%) were receiving proton pump inhibitors, as compared with $35(65 \%)$ of the 54 cases who did not have a relapse (unadjusted OR 5.2, 95\% CI 1.1-24.6). The only other factor associated with relapse in the univariate analysis was diabetes mellitus (unadjusted OR 2.7, 95\% CI 0.8-9.2). In the multivariate analysis, after adjustment for the presence of diabetes, use of proton pump inhibitors was the only fac-

\section{Table 3: Clinical characteristics of hospital patients with $C$. difficile diarrhea and matched} control subjects*

\begin{tabular}{|c|c|c|c|}
\hline \multirow[b]{2}{*}{ Characteristic } & \multicolumn{2}{|c|}{ Group; no. (\%) of patients† } & \multirow[b]{2}{*}{$\begin{array}{l}\text { Unadjusted } \\
\text { OR }(95 \% \mathrm{Cl})\end{array}$} \\
\hline & $\begin{array}{l}\text { Case group } \\
\quad n=94\end{array}$ & $\begin{array}{l}\text { Control group } \\
\quad n=94\end{array}$ & \\
\hline Age, mean (SD), yr & $75.5(13.4)$ & $73.0(11.2)$ & $p=0.17$ \\
\hline Female sex & $59(63)$ & $44(47)$ & $1.9(1.1-3.4)$ \\
\hline Institutionalized $\neq$ & $15(16)$ & $10(11)$ & $1.6(0.7-3.8)$ \\
\hline $\begin{array}{l}\text { Time from hospital admission to diagnosis } \\
\text { of } C \text {. difficile diarrhea, mean (median) }\end{array}$ & 22 (8) & - & - \\
\hline Length of hospital stay, mean (median) & - & $33(16)$ & - \\
\hline \multicolumn{4}{|l|}{ Comorbidities } \\
\hline Renal failure & $21(22)$ & $6 \quad(6)$ & $4.2(1.6-11.0)$ \\
\hline Diabetes mellitus & $22(23)$ & $19(20)$ & $0.8(0.4-1.6)$ \\
\hline Hypothyroidism & $19(20)$ & $15(16)$ & $1.3(0.6-2.8)$ \\
\hline Cancer & $17(18)$ & $29(31)$ & $0.5(0.2-1.0)$ \\
\hline MRSA infection & $20(21)$ & 4 (4) & $6.1(2.0-18.8)$ \\
\hline Charlson Comorbidity Index score $\mathrm{e}^{18}$ & $2.2(1.8)$ & $2.1(1.7)$ & $p=0.55$ \\
\hline Hospital admission in 3 mo before index admission & $36(38)$ & $16(17)$ & $3.0(1.5-6.0)$ \\
\hline \multicolumn{4}{|l|}{ Antibiotic exposure } \\
\hline No. of antibiotics, mean (SD) & $1.9(1.0)$ & $2.2(1.2)$ & $p=0.06$ \\
\hline Ampicillin or analogue & $5 \quad(5)$ & 7 (7) & $0.7(0.2-2.3)$ \\
\hline Clindamycin & $3(3)$ & $1(1)$ & $3.1(0.3-30.0)$ \\
\hline $\begin{array}{l}\text { Any second- or third-generation } \\
\text { cephalosporin }\end{array}$ & $9(9)$ & $7(7)$ & $1.3(0.5-3.7)$ \\
\hline Any quinolone§ & $57(61)$ & $55(58)$ & $1.1(0.6-1.9)$ \\
\hline High-risk antibiotic & $14(14)$ & $14(14)$ & $1.0(0.4-2.2)$ \\
\hline No antibiotic & 1 (1) & 1 (1) & $1.0(0.06-16.2$ \\
\hline 1 antibiotic & $37(39)$ & $32(34)$ & $1.3(0.7-2.3)$ \\
\hline 2 antibiotics & $28(30)$ & $25(27)$ & $1.2(0.6-2.2)$ \\
\hline$\geq 3$ antibiotics & $28(30)$ & $36(38)$ & $0.7(0.4-1.2)$ \\
\hline \multicolumn{4}{|l|}{ Acid suppressive therapy } \\
\hline $\mathrm{H}_{2}$ blocker & 1 (1) & $4 \quad(4)$ & $0.2(0.03-2.2)$ \\
\hline PPI & $60(64)$ & $34(36)$ & $3.1(1.7-5.6)$ \\
\hline PPI use $>6 \mathrm{mo}$ & $22(23)$ & $4 \quad(5)$ & $6.9(2.3-20.8)$ \\
\hline \multicolumn{4}{|l|}{ Outcome } \\
\hline Colectomy for severe colitis & $8 \quad(9)$ & - & - \\
\hline$\geq 1$ relapses & $21(22)$ & - & - \\
\hline $\begin{array}{l}\text { Admission to ICU because of } C \text {. difficile- } \\
\text { associated sepsis }\end{array}$ & $18(19)$ & - & - \\
\hline Acute renal failure requiring dialysis & $12(13)$ & $2(2)$ & $6.7(1.5-31.0)$ \\
\hline Death 9 & $21(22)$ & $12(14)$ & $2.0(0.9-4.3)$ \\
\hline
\end{tabular}

Note: SD = standard deviation, MRSA = methicillin-resistant Staphylococcus aureus, ICU = intensive care unit. *Patients were matched by age within 5 years, ward, class of antibiotics and, if possible, number of antibiotics. †Unless stated otherwise.

‡Long-term residence in a chronic care setting or hospital stay in an acute care setting for more than 2 months. $\S$ Patients received either levofloxacin or ciprofloxacin.

T Within 30 days after $C$. difficile diagnosis for cases; in the following 30 days for controls. 
tor significantly associated with risk of relapse (adjusted OR 5.1, 95\% CI 1.1-24.9) (data not shown in tabular form).

\section{Interpretation}

In this hospital-based study, patients with $C$. difficile diarrhea had substantial mortality and morbidity. We found that the use of proton pump inhibitors was independently associated with an increased risk of $C$. difficile diarrhea. We observed this association in the cohort study (which allowed us to adjust for some antibiotic-related confounding) as well as in the case-control study (which took place in another institution and allowed us to also control for other non-antibiotic-related confounding).

Ingestion of $C$. difficile can result in either excretion, asymptomatic colonization of the gut, or disease with diarrhea, colitis or pseudomembranous colitis. ${ }^{22}$ The normal stomach acidity is an important host defence against ingested pathogens and provides protection against enteric infections. ${ }^{7}$ We hypothesized that the decreased gastric acidity induced by the use of proton pump inhibitors increases the susceptibility of hospital patients to colonization and subsequent infection with $C$. difficile. Significant bacterial overgrowth and even colonization with fecal type bacteria ${ }^{6}$ has been demonstrated in the upper gastrointestinal tract of patients receiving acid suppressive therapy, ${ }^{7}$ with higher counts in patients taking proton pump inhibitors, ${ }^{6}$ presumably because these agents are more effective than $\mathrm{H}_{2}$ blockers at blocking gastric acid secretion.

An association between acid suppressive therapy and C. difficile diarrhea or colitis has been suggested in previous studies., ${ }^{4,10,11}$ None of these studies controlled for differences in antibiotic use, nor did they distinguish between different types of acid suppressive therapies. One study found a nonsignificant association between acid antisecretory therapy and C. difficile diarrhea, ${ }^{4}$ but the lack of statistical significance may have reflected limited power. A recent, brief report $^{3}$ supports our findings of an association between use of proton pump inhibitors and $C$. difficile diarrhea after adjustment for antibiotic exposure and receipt of chemotherapy.

Table 4: Factors associated with $C$. difficile diarrhea in case and control subjects (multivariate logistic regression)

\begin{tabular}{lcc}
\hline \multirow{2}{*}{ Characteristic } & \multicolumn{2}{c}{ Adjusted OR $(95 \% \mathrm{Cl})$} \\
\cline { 2 - 3 } & \multicolumn{1}{c}{ Model 1 } & Model 2 \\
\hline PPI use & $2.7(1.4-5.2)$ & $2.6(1.3-5.0)$ \\
Female sex & $2.1(1.1-4.0)$ & $2.5(1.2-5.0)$ \\
Prior renal failure & $4.3(1.5-11.9)$ & $5.1(1.7-15.2)$ \\
Hospital admission in 3 mo & & \\
$\quad$ before index admission & $2.6(1.4-5.2)$ & $2.9(1.4-6.1)$ \\
Cancer & $0.8(0.4-1.8)$ & $1.0(0.4-2.2)$ \\
MRSA colonization* & - & $7.8(2.3-26.0)$ \\
\hline
\end{tabular}

*MRSA colonization was excluded initially (model 1) because of a concern of detection bias. The number of antibiotics, exposure to high-risk antibiotics, the Charlson Comorbidity Index score and age were not significant factors and so were dropped from the final model and did not change estimates for PPI exposure.
In our case-control study, in addition to other clinical factors, we matched for type and number of antibiotics, because they are the most important known risk factors for C. difficile diarrhea.

Decreased gastric acidity has been associated with renal failure ${ }^{23}$ and older age, ${ }^{24}$ and it may be a factor contributing to the association of $C$. difficile diarrhea with renal failure and older age observed in our study, and in previous reports. ${ }^{22,25}$ Use of proton pump inhibitors has been associated with elevated gastrin levels, ${ }^{26}$ which have been shown to have trophic effects on the colonic mucosa. ${ }^{27}$ Proton pumps have also been described in the colon, ${ }^{28}$ but their function is unclear. We have postulated that decreased gastric acidity results in inadequate sterilization of ingested organisms, but other mechanisms are possible. Proton pump inhibitors may also contribute to the disruption of the bowel flora by allowing bacterial colonization of the stomach and upper small intestine; ${ }^{7}$ however, it is unclear what effect this might have on colonic flora. Use of proton pump inhibitors may then contribute significantly to outbreaks of C. difficile diarrhea by resulting in increased numbers of susceptible hosts as well as possibly increasing the numbers of carriers in the population.

In the cohort study, the effect of proton pump inhibitors on the risk of $C$. difficile diarrhea appeared to be greater among the patients who were taking low-risk antibiotics (e.g., cefazolin) than among those taking high-risk antibiotics. This difference suggests that the use of proton pump inhibitors may be an important effect modifier of antibiotic risk of $C$. difficile infection.

In our study we found many factors that suggest a significant association between the use of proton pump inhibitors and $C$. difficile diarrhea. These include time order (the cases were exposed to proton pump inhibitors before their symptoms developed) and dose response (the association was even stronger among patients with more prolonged exposure to proton pump inhibitors). In addition, our cohort study and our case-control study yielded similar findings despite the fact that they were conducted in different hospitals with different study designs. Furthermore, the proton pump inhibitor used in one hospital was primarily omeprazole, while in the other hospital it was pantoprazole, which suggests a class effect of these drugs. Our findings are also consistent with those from the study by Cunningham and colleagues. ${ }^{3}$ The hypothesis is also coherent with reduced gastric acidity being a risk factor for other infectious enteric diseases.

Almost $50 \%$ of the patients receiving antibiotics in our cohort study were prescribed proton pump inhibitors, with another $10 \%$ receiving $\mathrm{H}_{2}$ blockers. In the majority of the patients in the case-control study, we could not ascertain from the chart review why the patients were prescribed a proton pump inhibitor. A recent report suggested that acid suppressive therapy is overused in hospital patients and demonstrated that $46 \%$ of the patients in whom they determined the prescription unnecessary were still taking the medications 3 
months after discharge. ${ }^{29}$ Although concerns have been raised regarding overuse of proton pump inhibitors, and despite their high $\operatorname{cost}^{30}$ and the potential risks of prolonged achlorhydria,$^{14}$ the use of these drugs has been steadily increasing. Our data suggest that initiatives to curtail inappropriate use of proton pump inhibitors should be considered.

\section{Limitations}

We matched case and control subjects by type of antibiotics; however, this was particularly difficult for patients with longer hospital stays, who may have received several courses of antibiotics. Although the difference was not statistically significant, the control subjects tended on average to have had longer hospital stays and to have received more antibiotics than the cases. We tried to measure and adjust for most of the known risk factors for $C$. difficile diarrhea, but use of proton pump inhibitors may have been associated with some other unidentified risk factor, or with sicker patients who are perhaps more susceptible to $C$. difficile diarrhea. However, the case and control subjects had similar rates of death, Charlson Comorbidity Index scores and mean ages. We did not evaluate tube feeding as a risk factor, as less than $5 \%$ of the cases had been fed this way. $\mathrm{Al}-$ though the antitoxin assay is very sensitive, improper handling of the stool specimen may result in inactivation of the toxin. ${ }^{19}$ Exclusion of all patients with diarrhea who were negative for $C$. difficile toxin should have decreased the risk of misclassifying the control subjects, but it may have resulted in an underestimate of the incidence.

In conclusion, $C$. difficile diarrhea is associated with increased morbidity and mortality. Among the risk factors identified, the use of proton pump inhibitors may be an important, previously unrecognized and potentially modifiable risk factor for initial occurrence, and relapse. The use of these drugs should be evaluated carefully in hospital patients receiving antibiotics, especially in those with a diagnosis of $C$. difficile diarrhea.

\section{This article has been peer reviewed.}

From the Departments of Critical Care (Dial) and Respirology (Dial, Menzies), Montreal Chest Institute, the Departments of Critical Care (Dial), Biochemistry (Alrasadi) and Pharmacy (Manoukian), Sir Mortimer B. Davis Jewish General Hospital, and the Departments of Respirology (Dial, Menzies) and Geriatrics (Huang), Royal Victoria Hospital, Montréal, Que.

Competing interests: None declared.

Contributors: Sandra Dial drafted the manuscript, was responsible for the study concepts and designs and for data analysis and interpretation, and provided statistical expertise. Khalid Alrasadi provided study materials and patients and was responsible for data collection and assembly. Chantal Manoukian provided study materials and patients, and administrative and technical support. Allen Huang provided study materials and administrative and technical support and was responsible for data collection and assembly. Dick Menzies provided statistical expertise and administrative and technical support. All of the authors revised the draft critically for important intellectual content and approved the final version.

Acknowledgements: We thank Dr. Elizabeth MacNamara for providing suggestions and a resident to assist with the performance of the project, Sylvie Carle for providing antibiotic and acid suppressive therapy data from the pharmacy database used in the study, and infection control nurses Guylaine Morin, Pearl Orenstein, Barbara Amihod and Norma Consolacion for their help in providing the data on the cases.

\section{References}

1. Bartlett JG. Clinical practice. Antibiotic-associated diarrhea. $N$ Engl $7 \mathrm{Med}$ 2002;346(5):334-9.

2. Morris A, Jobe B, Stoney M, Deveney C, Deveney K. Clostridium difficile colitis: an increasingly aggressive iatrogenic disease: (23). Dis Colon Rectum 2001; 44(4):A5-A26.

3. Cunningham R, Dale B, Undy B, Gaunt N. Proton pump inhibitors as a risk factor for Clostridium difficile diarrhoea. 7 Hosp Infect 2003;54(3):243-5.

4. Kyne L, Warny M, Qamar A, Kelly CP. Asymptomatic carriage of Clostridium difficile and serum levels of IgG antibody against toxin A. N Engl f Med 2000; 342(6):390-7.

5. Kyne L, Hamel MB, Polavaram R, Kelly CP. Health care costs and mortality associated with nosocomial diarrhea due to Clostridium difficile. Clin Infect Dis 2002;34(3):346-53.

6. Thorens J, Froehlich F, Schwizer W, Saraga E, Bille J, Gyr K, et al. Bacterial overgrowth during treatment with omeprazole compared with cimetidine: a prospective randomised double blind study. Gut 1996;39(1):54-9.

7. Williams C. Occurrence and significance of gastric colonization during acidinhibitory therapy. Best Pract Res Clin Gastroenterol 2001;15(3):511-21.

8. Gurian L, Ward TT, Katon RM. Possible foodborne transmission in a case of pseudomembranous colitis due to Clostridium difficile: influence of gastrointestinal secretions on Clostridium difficile infection. Gastroenterol 1982;83(2):465-9.

9. Bignardi GE. Risk factors for Clostridium difficile infection. 7 Hosp Infect 1998; 40(1):1-15.

10. Simor AE, Yake SL, Tsimidis K. Infection due to Clostridium difficile among elderly residents of a long-term-care facility. Clin Infect Dis 1993;17(4):672-8.

11. Walker KJ, Gilliland SS, Vance-Bryan K, Moody JA, Larsson AJ, Rotschafer $\mathrm{JC}$, et al. Clostridium difficile colonization in residents of long-term care facilities: prevalence and risk factors. 7 Am Geriatr Soc 1993;41(9):940-6.

12. Archimandritis A, Souyioultzis S, Katsorida M, Tzivras M. Clostridium difficile colitis associated with a "triple" regimen, containing clarithromycin and metronidazole, to eradicate Helicobacter pylori. 7 Intern Med 1998;243(3):251-3.

13. Rai R, Rai S. Pseudomembranous colitis requiring surgical intervention following triple therapy for Helicobacter pylori eradication. ANZ 7 Surg 2002;72(12):917-9.

14. Laine L, Ahnen D, McClain C, Solcia E, Walsh JH. Review article: potential gastrointestinal effects of long-term acid suppression with proton pump inhibitors. Aliment Pharmacol Ther 2000;14(6):651-68.

15. Wistrom J, Norrby SR, Myhre EB, Eriksson S, Granstrom G, Lagergren L, et al. Frequency of antibiotic-associated diarrhoea in 2462 antibiotic-treated hospitalized patients: a prospective study. 7 Antimicrob Chemother 2001;47(1):43-50.

16. Pothoulakis C, Lamont JT. Clostridium difficile colitis and diarrhea. Gastroenterol Clin North Am 1993;22(3):623-37.

17. Bennett RG, Greenough WB III. Approach to acute diarrhea in the elderly. Gastroenterol Clin North Am 1993;22(3):517-33.

18. Charlson ME, Pompei P, Ales KL, MacKenzie CR. A new method of classifying prognostic comorbidity in longitudinal studies: development and validation. 7 Chronic Dis 1987:40(5):373-83.

19. Wilkins TD, Lyerly DM. Clostridium difficile testing: after 20 years, still challenging. 7 Clin Microbiol 2003;41(2):531-4.

20. Kleinbaum DG. Logistic regression: a self-learning text. New York: Springer; 1994

21. Kelly CP, Lamont JT. Clostridium difficile infection. Annu Rev Med 1998;49:375-90.

22. Wilcox MH. Clostridium difficile infection and pseudomembranous colitis. Best Pract Clin Gastroenterol 2003;17(3):475-93.

23. Muto S, Murayama N, Asano Y, Hosoda S, Miyata M. Hypergastrinemia and achlorhydria in chronic renal failure. Nephron 1985;40(2):143-8.

24. Hurwitz A, Brady DA, Schaal SE, Samloff IM, Dedon J, Ruhl CE. Gastric acidity in older adults. 7AMA 1997;278(8):659-62.

25. Safdar N, Maki DG. The commonality of risk factors for nosocomial colonization and infection with antimicrobial-resistant Staphylococcus aureus, enterococcus, gram-negative bacilli, Clostridium difficile, and Candida. Ann Intern Med 2002;136(11):834-44.

26. Ligumsky M, Lysy J, Siguencia G, Friedlander Y. Effect of long-term, continuous versus alternate-day omeprazole therapy on serum gastrin in patients treated for reflux esophagitis. 7 Clin Gastroenterol 2001;33(1):32-5.

27. Klingensmith ME, Neville LJ, Delpire E, Wolfe MM, Soybel DI. Gastrin-mediated effects of omeprazole on rat colon mucosa. Surgery 1999;126(2):272-8.

28. Mukherjee S. Diarrhea associated with lansoprazole. 7 Gastroenterol Hepatol 2003;18(5):602-3.

29. Parente F, Cucino C, Gallus S, Bargiggia S, Greco S, Pastore L, et al. Hospital use of acid-suppressive medications and its fallout on prescribing in general practice: a 1 month survey. Aliment Pharmacol Ther 2003;17(12):1503-6.

30. Westbrook JI, Duggan AE, McIntosh JH. Prescriptions for antiulcer drugs in Australia: volume, trends, and costs. BMF 2001;323(7325):1338-9.

Correspondence to: Dr. Sandra Dial, Montreal Chest Institute, 3650 Saint-Urbain, Rm. K1.14, Montréal QC H2X 2P4; fax 514 843-2083; sandra.dial@mcgill.ca 